\title{
Control of Power Distribution for Multiple Receivers in SIMO Wireless Power Transfer System
}

\author{
Gunyoung Kim · Seunghyun Boo $\cdot$ Sanghoek Kim $\cdot$ Bomson Lee
}

\begin{abstract}
A method to control the power distribution among receivers by the load values in a single-input, multiple-output (SIMO) wireless power transfer (WPT) system is investigated. We first derive the value of loads to maximize total efficiency. Next, a simple, but effective analytical formula of the load condition for the desired power distribution ratio is presented. The derived load solutions are simply given by system figure of merits and desired power ratios. The formula is validated with many numerical examples via electromagnetic simulations. We demonstrate that with the choice of loads from this simple formula, the power can be conveniently and accurately distributed among receivers for most practical requirements in SIMO WPT systems.
\end{abstract}

Key Words: Optimum Load, Power Distribution, Single-Input Multi-Output, Transfer Efficiency, Wireless Power Transfer.

\section{INTRODUCTION}

A magnetically coupled wireless power transfer (WPT) system consisting of one transmitter and one receiver was successfully demonstrated using coupled-mode theory (CMT) in [1]. The WPT technology has since been studied to enable various applications, such as charging mobile phones, tablets, notebooks, home appliances, motor vehicles, and biomedical devices. The ongoing goal of researchers in this field is to achieve a higher efficiency over a longer distance. The use of an optimum load to realize the upper limit of transfer efficiencies was highlighted from the beginning [1] and has been one important design guideline in practice. To enhance the WPT efficiency beyond the upper limit, some WPT systems using relay coils and metamaterial slabs have been studied [2-4].

Recently, the research interest in WPT technology has been extended to accommodate multiple-input multiple-output (MIMO) systems. In particular, the MIMO WPT [5] system has been attracting attention as a way to enable the energy autono- my of Internet of Things (IoT) sensors. For general MIMO WPT systems, the efficiencies are usually evaluated numerically using a $\mathrm{Z}$-matrix circuit formulation [6]. The loads to achieve a specifically required power distribution to multiple receivers can be found numerically using a genetic algorithm. However, the exact analytic (or closed-form) solutions for them have not yet been found for a general MIMO system.

A single-input multiple-output (SIMO) system can be considered a subset of MIMO systems. The overall efficiency of a SIMO WPT system was initially examined using CMT [7]. A circuit-centric matrix analytical model that predicts the behavior of SIMO systems was also presented [8].

In addition to achieving high efficiency, power distribution among receivers has been of great interest in designing a SIMO WPT system. For example, [9] proposed a time-shared charging technique to distribute power to receivers. In an effort to reduce the coupling effects between receivers, [10] diversified the resonant frequencies among the receivers. In this method, the power division among receivers was still controlled by ad-

Manuscript received February 8, 2018 ; Accepted May 22, 2018 ; Accepted June 19, 2018. (ID No. 20180208-015J)

Department of Electronics Engineering, Kyung Hee University, Yongin, Korea.

*Corresponding Author: Bomson Lee (e-mail: bomson@khu.ac.kr)

This is an Open-Access article distributed under the terms of the Creative Commons Attribution Non-Commercial License (http://creativecommons.org/licenses/by-nc/4.0) which permits unrestricted non-commercial use, distribution, and reproduction in any medium, provided the original work is properly cited.

(c) Copyright The Korean Institute of Electromagnetic Engineering and Science. All Rights Reserved. 
justing the charging time. The methods proposed in $[11,12]$ were presented in a relatively complex and abstract form to be used in design practice.

Another method to control the power distribution among receivers is to adjust the loads at the receivers. In [13], a power division was achieved by utilizing impedance inverters at the receiver sides on a two-receiver example. In [14], an impedance matching method for the power distribution among receivers was proposed based on coupled-mode and circuit theories. The actual power distribution among receivers, however, differs greatly from the desired distribution, especially in a condition of highly asymmetric distribution. [15] developed a new power control scheme by combining maximum efficiency point tracking (MEPT) with time-division multiplexing (TDM). In [16], multiple-receiver WPT-based battery voltage equalization was investigated using analytical derivations and experiments.

In this paper, we derive and present a simple analytic solution to control the power distributions among multiple receivers. The necessary receiver load values for a desired power distribution can be easily computed using the presented solution with little loss of accuracy. The proposed method of realizing arbitrarily desired power distributions is verified using numerical examples in circuit and electromagnetic (EM) simulations.

\section{MODELING OF A SIMO WPT SYSTEM}

\section{Formulation of a SIMO WPT System}

Fig. 1(a) and (b) show a typical SIMO WPT system and its equivalent circuit. The system is assumed to have a single transmitter and multiple receivers. All the loops (or coils) have some inductances $\left(L_{i}\right)$ and resistances $\left(R_{i}\right)$. Each coil is loaded with a capacitor $\left(C_{i}\right)$ for resonance at a specific design frequency. The transmitter is excited by a voltage source $\left(V_{0}\right)$, and the $N$ receivers have load resistances $\left(R_{L i}\right)$ to receive transferred powers. Once the sizes and positions of the transmitter and receivers are specified, the coupling coefficients between any two loops can be determined using the ratio of magnetic flux lines from one loop to the other [17]. The WPT system can then be analyzed in terms of the efficiencies for each receiver and for the total system. For $(N+1)$ loops of the SIMO WPT system in Fig. 1, we can write $(N+1)$ equations using KVL. They can be rearranged using a $\mathrm{Z}$-matrix formulation. The $\mathrm{Z}$-matrix as a function frequency is given by:

$$
\left[\begin{array}{c}
V_{0} \\
0 \\
0 \\
\vdots \\
0
\end{array}\right]=\left[\begin{array}{ccccc}
Z_{00} & Z_{01} & Z_{02} & \cdots & Z_{0 N} \\
Z_{10} & Z_{11} & Z_{12} & \cdots & Z_{1 N} \\
Z_{20} & Z_{21} & Z_{22} & \cdots & Z_{2 N} \\
\vdots & \vdots & \vdots & \vdots & \vdots \\
Z_{N 0} & Z_{N 1} & Z_{N 2} & \cdots & Z_{N N}
\end{array}\right]\left[\begin{array}{c}
I_{0} \\
I_{1} \\
I_{2} \\
\vdots \\
I_{N}
\end{array}\right] .
$$

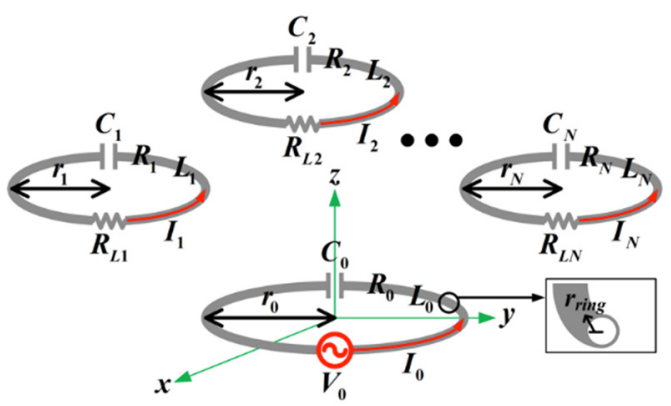

(a)

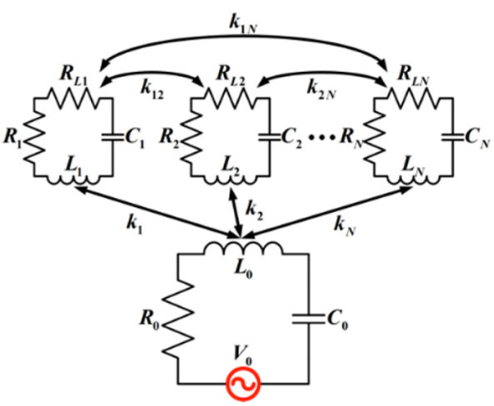

(b)

Fig. 1. A general configuration of a SIMO WPT system with one transmitter and $N$ receivers. (a) Geometry and (b) equivalent circuit.

In (1), $V_{0}$ in the column matrix [ $\left.V\right]$ is the supplied voltage of the transmitter. The principal diagonal elements of $[Z]$ at the resonant design frequency are given by:

$$
Z_{00}=R_{0}
$$

and

$$
Z_{i i}=R_{i}+R_{L i}(\text { for } i=1,2, \cdots, N),
$$

where $R_{i} \mathrm{~s}$ are the loop resistances and $R_{L i}$ is are the load resistances for the receivers. The other elements of $[Z]$ are given by:

$$
Z_{i j}=Z_{j i}=-j \omega_{0} k_{i j} \sqrt{L_{i} L_{j}} \quad(i \neq j \text { for } i, j=0,1,2, \cdots, N),
$$

where $k_{i s}$ are the coupling coefficients between two loops. When all the elements of the $Z$-matrix are given, the current on each loop is determined by $[I]=[Z]^{-1}[\mathrm{~V}]$, in which all couplings between any two loops including the mutual are all considered. The input power and the received power at each receiver can then be evaluated using:

$$
\begin{aligned}
& P_{i n}=\frac{1}{2} \operatorname{Re}\left[V_{0} I_{0}^{*}\right] \\
& =\frac{1}{2}\left|I_{0}\right|^{2} R_{0}+\frac{1}{2} \sum_{i=1}^{N}\left|I_{i}\right|^{2}\left(R_{i}+R_{L i}\right) \quad(\text { for } i=1,2, \cdots, N)
\end{aligned}
$$

and

$$
P_{L i}=\frac{1}{2}\left|I_{i}\right|^{2} R_{L i} \quad(\text { for } i=1,2, \cdots, N) .
$$




\section{Derivation of the Optimum Load and Efficiency in SIMO}

The efficiency for the $i^{\text {th }}$ receiver at the resonant frequency is defined and expressed as:

$$
\begin{aligned}
\eta_{i}=\frac{P_{L i}}{P_{i n}}= & \frac{\frac{1}{2}\left|I_{i}\right|^{2} R_{L i}}{\frac{1}{2}\left|I_{0}\right|^{2} R_{0}+\frac{1}{2} \sum_{i=1}^{N}\left|I_{i}\right|^{2}\left(R_{i}+R_{L i}\right)} \\
& =\frac{\left|\frac{I_{i}}{I_{0}}\right|^{2} \frac{R_{L i}}{R_{0}}}{1+\sum_{i=1}^{N}\left|\frac{I_{i}}{I_{0}}\right|^{2}\left(\frac{R_{i}+R_{L i}}{R_{0}}\right)} .
\end{aligned}
$$

The efficiency can be evaluated using (7) or by $\left|S_{\mathrm{i} 0}\right|^{2} /\left(1-\left|S_{00}\right|^{2}\right)$ using EM simulations. The efficiencies using the two methods always agree well. If the coupling coefficients between any two receivers shown in Fig. 1 are negligible $\left(k_{i j} \rightarrow 0, i \neq j\right.$ for $i, j=1$, $2, \cdots, N)$, the ratio of the currents on the $i^{\text {th }}$ receiver and the transmitter is expressed in an analytic form, as in [18]:

$$
\frac{I_{i}}{I_{0}}=\frac{j \omega k_{i} \sqrt{L_{i} L_{0}}}{R_{i}+R_{L i}}
$$

and (7) can be simplified to:

$$
\eta_{i}\left(\beta_{1}, \beta_{2}, \cdots, \beta_{N}\right)=\frac{\frac{F_{i}^{2} \beta_{i}}{\left(1+\beta_{i}\right)^{2}}}{1+\sum_{i=1}^{N} \frac{F_{i}^{2}}{\left(1+\beta_{i}\right)}}
$$

where $F_{i}$ and $\beta_{i}$ are defined as the figure of merit and the normalized load resistance defined by:

$$
F_{i}=k_{i} \sqrt{Q_{i} Q_{0}}=k_{i} \omega_{0} \sqrt{\frac{L_{i} L_{0}}{R_{i} R_{0}}}
$$

and

$$
\beta_{i}=\frac{R_{L i}}{R_{i}}
$$

where $k_{i}$ is the coupling coefficient between the transmitter and $i^{\text {th }}$ receiver [17], and $Q_{i}$ is the quality factor of the $i^{\text {th }}$ receiver.

The figure of merit (10) is entirely determined by the specification of the $\mathrm{Tx}$ and $\mathrm{Rx}$ loops and the arrangement between them and is not dependent on the load $R_{L i}$. The system efficiency (or total efficiency) is defined and given by:

$$
\eta_{t}\left(\beta_{1}, \beta_{2}, \cdots, \beta_{N}\right)=\frac{\sum_{i=1}^{N} P_{L i}}{P_{i n}}=\sum_{i=1}^{N} \eta_{i}=\frac{\sum_{i=1}^{N} \frac{F_{i}^{2} \beta_{i}}{\left(1+\beta_{i}\right)^{2}}}{1+\sum_{i=1}^{N} \frac{F_{i}^{2}}{\left(1+\beta_{i}\right)}}
$$

as a function of $N$ normalized loads.

To derive $\beta_{i}$ s $(i=1,2, \cdots, N)$, which maximize $\eta_{t}$ in (12), we require:

$$
\frac{\partial \eta_{t}}{\partial \beta_{i}}=0 \text { for } i=1,2, \cdots, N
$$

The $N$ partial differential equations must be solved simultaneously. The normalized load resistance $\beta_{i}$ for $i=1,2, \cdots, N$ satisfying (13) turned out to be identical as a constant $\beta_{\text {opt }}$ for all receivers [11], although the figures of merit $\left(F_{i}\right)$ given by $(10)$ are different. They are:

$$
\beta_{1}=\beta_{2}=\cdots=\beta_{N}=\sqrt{1+\sum_{i=1}^{N} F_{i}^{2}}=\beta_{o p t} .
$$

It is convenient if we define the overall figure of merit of a SIMO system $(F)$ as:

$$
F=\sqrt{\sum_{i=1}^{N} F_{i}^{2}}
$$

The normalized optimum load $\beta_{\text {opt }}$ in (14) can then be simply expressed as:

$$
\beta_{o p t}=\sqrt{1+F^{2}}
$$

With (16), the maximum of $\eta_{t}$ in (12) can be expressed as:

$$
\begin{aligned}
& \eta_{t}\left(\beta_{\text {opt }}\right)=\sum_{i=1}^{N} \eta_{i}\left(\beta_{\text {opt }}\right) \\
& =\frac{\sum_{i=1}^{N} F_{i}^{2}}{\left(1+\sqrt{1+F^{2}}\right)^{2}}=\frac{F^{2}}{\left(1+\sqrt{1+F^{2}}\right)^{2}}
\end{aligned}
$$

after some algebraic arrangements. Notice that (16) and (17) have exactly the same forms as those for SISO systems [16]. Note that as $F(15)$ becomes very large, the efficiency in (17) goes to 1 . As $F$ becomes very small, the efficiency goes to zero.

3. Numerical Examples with One Transmitter and Two Receivers $(N=2)$ As an example, assume that we have one $\mathrm{Tx}$ and two $\mathrm{Rx}$ loops, where the figures of merit between the $\mathrm{Tx}$ and $\mathrm{Rx}$ loops are $F_{1}=16.2$ and $F_{2}=10.7$. The efficiency of individual $\mathrm{Rx} \eta_{i}$ and the total system efficiency $\eta_{t}$ can be controlled by the (normalized) load $\beta_{i}$. From Fig. 2, we can analyze how selectively power can be distributed to each $\mathrm{Rx}$ in the following scenarios.

In the first scenario, let us suppose we want to deliver the most power to $\mathrm{Rx} 1$ instead of $\mathrm{Rx} 2$. Intuitively, one can achieve this by opening the load of $\operatorname{Rx} 2\left(\beta_{2} \rightarrow \infty\right)$ to suppress any power flow into $\mathrm{Rx} 2$ and re-direct that power to $\mathrm{Rx} 1$. Simultaneously, 
the load value of $\mathrm{Rx} 1$ should be optimized to receive the maximum power. This conjecture is consistent with the result in Fig. 2. Explicitly, when $\beta_{1}$ is about 16.2 and $\beta_{2} \rightarrow \infty, \eta_{1}$ is maximized to $88.4 \%$ and $\eta_{2}$ decreases to zero in Fig. $2(\mathrm{a})$ and (b).

The opposite is true in the second scenario in which we want to deliver the most power to $\mathrm{Rx} 2$ instead of Rx1. Fig. 2 shows that when $\beta_{1} \rightarrow \infty$ (Rx1 is open) and $\beta_{2}$ is about $10.7, \eta_{1}$ and $\eta_{2}$ reach zero and $82.9 \%$, respectively. Obviously, the maximum $\eta_{1}$ (88.4\%) is greater than the maximum $\eta_{2}(82.9 \%)$ because $\mathrm{Rx} 1$ is inherently more strongly coupled to $\mathrm{Tx}$ than $\mathrm{Rx} 2 ; F_{1}(=16.2)$ is greater than $F_{2}(=10.7)$.

Next, one may choose to maximize the total system efficiency $\eta_{t}$. The maximum total efficiency in Fig. 2(c) is about $90.2 \%$ when $\beta_{1}=\beta_{2}=\beta_{\text {opt }}=\sqrt{1+F^{2}}=\sqrt{1+16.2^{2}+10.7^{2}}=19.4$.

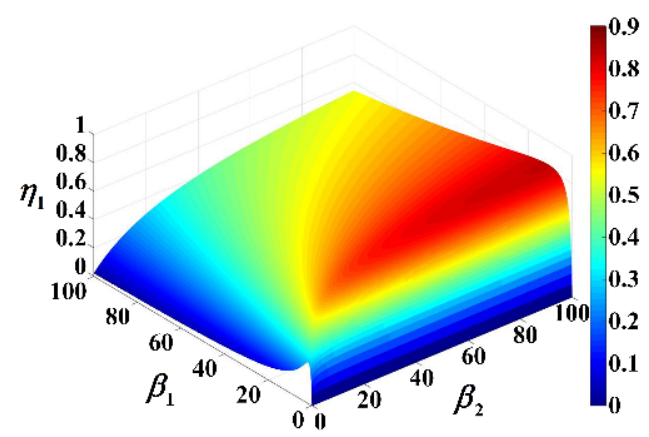

(a)

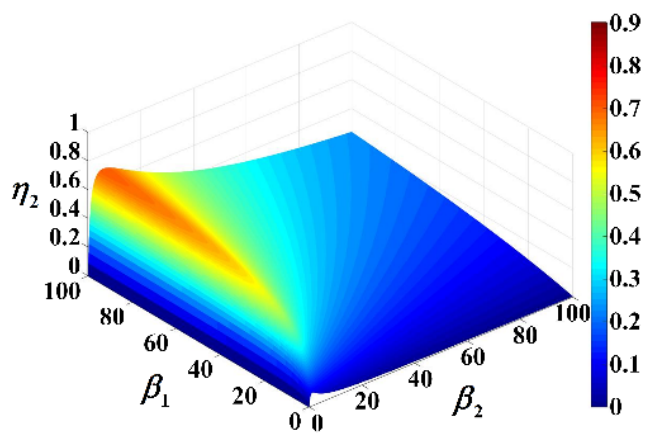

(b)

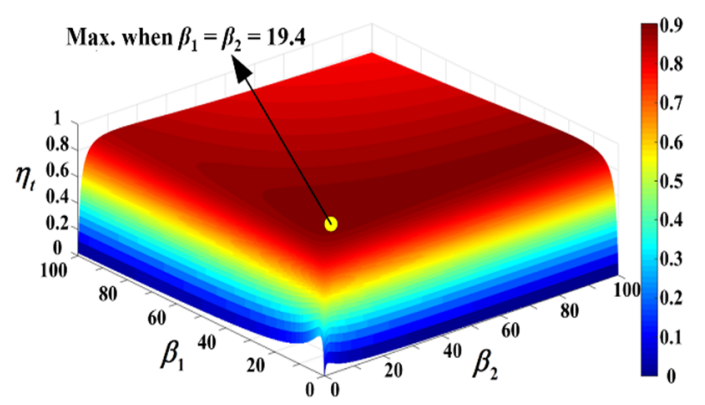

(c)

Fig. 2. Efficiencies as a function of the normalized load resistances $\beta_{1}$ and $\beta_{2}$ in the SIMO system $\left(N=2, F_{1}=16.2\right.$, and $F_{2}=$ 10.7). (a) Efficiency for the 1 st receiver $\left(\eta_{1}\right)$, (b) efficiency for the 2 nd receiver $\left(\eta_{2}\right)$, and (c) total efficiency of the SIMO system $\left(\eta_{t}\right)$.
When the total efficiency is maximized, the power is distributed to $\mathrm{Rx} 1$ and $\mathrm{R} \times 2$, with efficiency of $\eta_{1}=63.1 \%$ and $\eta_{2}=27.1 \%$.

Another important observation from Fig. 2(c) is that over a wide region of $\beta_{1}$ and $\beta_{2}$, the total efficiency $\eta_{t}$ is nearly invariant from the maximum of $90.2 \%$. This implies the power can be distributed among multiple receivers in almost any ratio users desire, with minimum loss in total efficiency.

\section{CONTROL OF POWER DiSTRIBUTION RATIO}

The choice of power delivery distribution is not limited to the three scenarios mentioned above in Section II-C. The continuous distribution of efficiencies in Fig. 2 suggests that users are allowed an infinite number of choices of power distribution to each $\mathrm{Rx}$, with total efficiency nearly unchanged. In this section, we propose a simple method to determine the load resistances of receivers to control the power distribution ratio. We also demonstrate the method using numerical examples.

\section{Load Resistance for Desired Power Distribution}

To demonstrate the method, we first define the power distribution ratio $\left(\alpha_{1}, \alpha_{2}, \cdots, \alpha_{N}\right)$ for the receivers as follows:

$$
\alpha_{i}=\frac{\eta_{i}}{\eta_{t}}=\frac{\frac{F_{i}^{2} \beta_{i}}{\left(1+\beta_{i}\right)^{2}}}{\sum_{i=1}^{N} \frac{F_{i}^{2} \beta_{i}}{\left(1+\beta_{i}\right)^{2}}} \text { for } i=1,2, \cdots N .
$$

Clearly, $\sum_{i=1}^{N} \alpha_{i}=1$. When $F_{i}$ s (for $i=1,2, \cdots, N$ ) are all known, $\alpha_{i}$ is determined by the choices of $\beta_{i}$ (for $i=1,2, \cdots, N$ ). In particular, when $\beta_{i}=\beta_{\text {opt }}$ (for $\left.i=1,2, \cdots, N\right)$ (14), $\alpha_{i}$ in (18) becomes:

$$
\alpha_{i, o p t}=\frac{\eta_{i}\left(\beta_{o p t}\right)}{\eta_{t}\left(\beta_{o p t}\right)}=\frac{\frac{F_{i}^{2} \beta_{o p t}}{\left(1+\beta_{o p t}\right)^{2}}}{\sum_{i=1}^{N} \frac{F_{i}^{2} \beta_{o p t}}{\left(1+\beta_{o p t}\right)^{2}}}=\frac{F_{i}^{2}}{\sum_{i=1}^{N} F_{i}^{2}}=\frac{F_{i}^{2}}{F^{2}},
$$

where $\alpha_{i, p \phi t}$ 's (for $i=1,2, \cdots, N$ ) are the optimum power distribution ratio to maximize the total efficiency (12). If the desired power distribution ratio $\left(\alpha_{i}^{\prime}\right.$ s for $\left.i=1,2, \cdots, N\right)$ is different from the optimum power distribution ratio $\left(\alpha_{i, o p t}\right.$ s for $\left.i=1,2, \cdots, N\right)$, the total efficiency may become somewhat lower than the maximum depending on the degree of the difference of the optimum (12).

In case a desired power distribution ratio $\left(\alpha_{i}^{\prime} \mathrm{s}\right)$ is specified first in (18), the load values, $\beta_{i}$ s, of the receivers can be obtained numerically. However, the exact analytic solution in a simple form has not been found yet. 


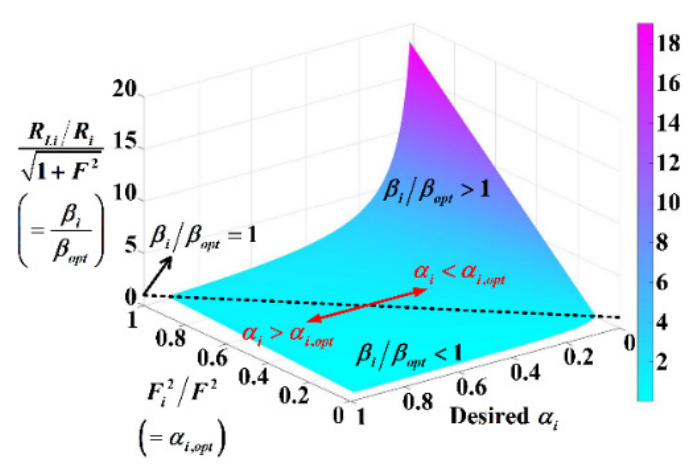

Fig. 3. Normalized load $\left(R_{L i} / R_{i}\right) / \sqrt{1+F^{2}} \quad\left(=\beta_{i} / \beta_{o p t}\right)$ as a function of desired $\alpha_{i}$ and $F_{i}^{2} / F^{2}\left(=\alpha_{i, o p t}\right)\left(0.05 \leq \alpha_{i}\right.$ and $F_{i}^{2} / F^{2}\left(=\alpha_{i, o p t}\right)$ $\leq 0.95)$.

Instead, we propose a simple, approximate solution to control the power distribution ratio. We first note that the total efficiency is insensitive to the choice of load resistances from Fig. 2(c) unless they deviate significantly from their optimum (16). Based on this observation, the ratio of (18) and (19) can be approximated as:

$$
\frac{\alpha_{i}}{\alpha_{i, o p t}} \approx \frac{\frac{F_{i}^{2} \beta_{i}}{\left(1+\beta_{i}\right)^{2}}}{\frac{F_{i}^{2} \beta_{o p t}}{\left(1+\beta_{o p t}\right)^{2}}}=\frac{\frac{\beta_{i}}{\left(1+\beta_{i}\right)^{2}}}{\frac{\beta_{o p t}}{\left(1+\beta_{o p t}\right)^{2}}} \text {. }
$$

We further assume $1+\beta \approx \beta$ (or $\beta>>1$ ), which is indeed the case for most practical WPT problems. This leads to the conclusion that to achieve the desired $\alpha_{i}, \beta_{i}$ is:

$$
\beta_{i}=\frac{R_{L i}}{R_{i}}=\beta_{o p t} \frac{\alpha_{i, o p t}}{\alpha_{i}}=\sqrt{1+F^{2}} \frac{F_{i}^{2} / F^{2}}{\alpha_{i}}
$$

for $i$ from 1 to $N$. The receiver load solution (21) can be used as a formula determined by only $F(15), F_{i}(10)$, and the desired $\alpha_{i}$. In Sections III-B and III-C, we will show that the accuracy of (21) is high enough for most practical problems unless the desired power distribution ratio $\left(\alpha_{1}, \alpha_{2}, \cdots, \alpha_{N}\right)$ deviates too much from its optimum $\left(F_{1}^{2} / F^{2}, F_{2}^{2} / F^{2}, \cdots, F_{N}^{2} / F^{2}\right)(19)$.

In Fig. 3, based on (21), we plot $\left(R_{L i} / R_{i}\right) / \sqrt{1+F^{2}}$ (or $\left.\beta_{i} / \beta_{\text {opt }}\right)$ as a function of the desired $\alpha_{i}$ and $F_{i}^{2} / F^{2}\left(=\alpha_{i, o p t}\right)$. With this universal curve, we can determine the normalized load resistance $\beta_{i}$ (or $R_{L i} / R_{i}$ ) to realize the desired $\alpha_{i}$ (for $i=1,2, \cdots, N$ ) once the figures of merit $\left(F_{i} \mathrm{~s}\right)$ for any specific SIMO WPT system are known.

For example, when $F_{1}=16.2$ and $F_{2}=F_{3}=11.4, F=22.9$ using (15), $\alpha_{1, o p t}=F_{1}^{2} / F^{2}=0.5, \alpha_{2, o p t}=\alpha_{3, o p t}=0.25$ from (19), $\beta_{o p t}=$ 22.9 from (14) or (16), and $\eta_{t}\left(\beta_{o p t}\right)=91.6 \%$ from (17). If the desired $\alpha_{1}, \alpha_{2}$, and $\alpha_{3}$ are $0.7,0.2$, and 0.1 , we can obtain $\beta_{1}=$
16.4, $\beta_{2}=28.6$, and $\beta_{3}=57.3$ using (21) and $\eta_{t}=91 \%$ using (12).

\section{Numerical Validation by Circuit Simulations $(N=2)$}

The proposed power distribution method was validated using circuit simulations. We physically have one $\mathrm{Tx}$ loop and two $\mathrm{Rx}$ loops, and the resonant frequencies of all of them are set to 6.78 $\mathrm{MHz}$. The circuit element values $(R, L$, and $C)$ are $0.034 \Omega$, $0.533 \mu \mathrm{H}$, and $1.034 \mathrm{nF}$ for the Tx loop and $0.017 \Omega, 0.223 \mu \mathrm{H}$, and $2.472 \mathrm{nF}$ for the Rx loop. The quality factors of the $\mathrm{Tx}$ and Rx loops are 668 and 559, respectively.

The coupling coefficients between the $\mathrm{Tx}$ and each $\mathrm{Rx}\left(k_{1}\right.$ and $k_{2}$ ) are assumed to be identical at 0.0265 ; therefore, $F_{1}=F_{2}$ $=16.2$ using (10). Fig. 4(a) shows the realized (or achieved) power distribution ratios and the total efficiencies as a function of the desired $\alpha_{1}$. Note that $\alpha_{2}=1-\alpha_{1}$.

The optimum power distribution ratio is $\alpha_{1, \text { opt }}=\alpha_{2, o p t}=0.5$ (= $16.2^{2} /\left(16.2^{2}+16.2^{2}\right)$ ) from (19). When $\beta_{1}=\beta_{2}=\beta_{\text {opt }}=22.9$ (16), the total efficiency is maximized to $\eta_{t}\left(\beta_{\text {opt }}\right)$ of $91.64 \%$. One can find that the power is distributed to $\mathrm{Rx} 1$ with an accurate ratio as we target. Fig. 4(a) also demonstrates that the closed-form formula (21) is more accurate than the formula presented in

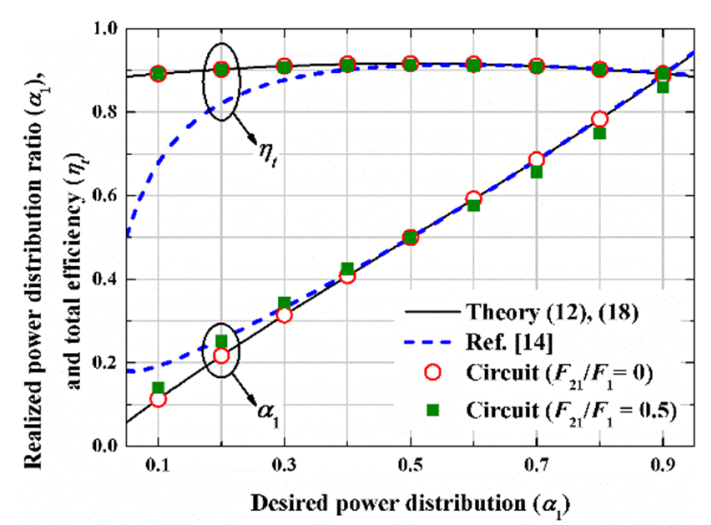

(a)

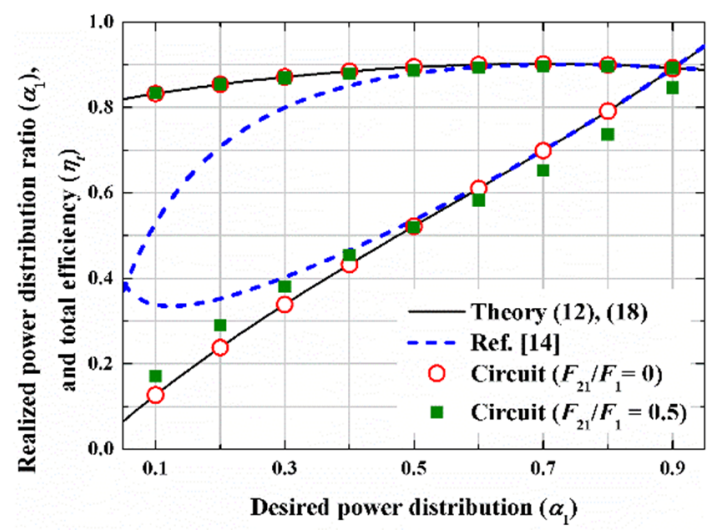

(b)

Fig. 4. Realized $\alpha_{1}$ and $\eta_{t}$ as a function of desired $\alpha_{1}\left(f_{0}=6.78 \mathrm{MHz}\right.$ and $N=2)$. (a) $\alpha_{1, o p t}=F_{1}^{2} / F^{2}=0.5=\alpha_{2, o p t}\left(F_{1}=F_{2}=16.2\right)$ and (b) $\alpha_{1, o p t}=F_{1}^{2} / F^{2}=0.7$ and $\alpha_{2, o p t}=0.3\left(F_{1}=16.2\right.$ and $F_{2}=$ 10.7). 
[14]. Furthermore, we can see that even when the mutual coupling between two receivers is considered with $F_{21} / F_{1}=0.5$, the results do not show much difference.

As an asymmetric example, the coupling coefficients between the $\mathrm{Tx}$ and each $\mathrm{Rx}\left(k_{1}\right.$ and $\left.k_{2}\right)$ are assumed to be 0.0265 and 0.0175. Therefore, $F_{1}$ and $F_{2}$ are 16.2 and 10.7. The realized power distribution ratios and the total efficiencies are shown in Fig. 4(b) as a function of the desired $\alpha_{1}$. The optimum power distribution ratio at $\operatorname{Rx} 1\left(\alpha_{1, p p t}\right)$ is $0.7 \mathrm{using}(19)$. When $\beta_{1}=\beta_{2}=$ $\beta_{\text {opt }}=19.4$, the total efficiency is maximized to $90.21 \%$. Unlike the first example, the realized power distribution ratio slightly deviates from the target ratio, especially when the target power distribution ratio is low. For example, when the target ratios $\alpha_{1}$ and $\alpha_{2}$ are 0.3 and 0.7 , the realized ratios are about 0.34 and 0.66 .

In Fig. 5, the realized power distribution ratios and total efficiencies are shown as a function of desired $\alpha_{1}$ for various maximum total efficiencies of $0.99,0.9,0.8$, and 0.7 (17) obtained when the overall figures $(F)$ of merit are 199, 19, 8.9, and 5.6, respectively. It is assumed that $N=2$ and $F_{1}^{2} / F^{2}=\alpha_{1, p p}=0.7$ for

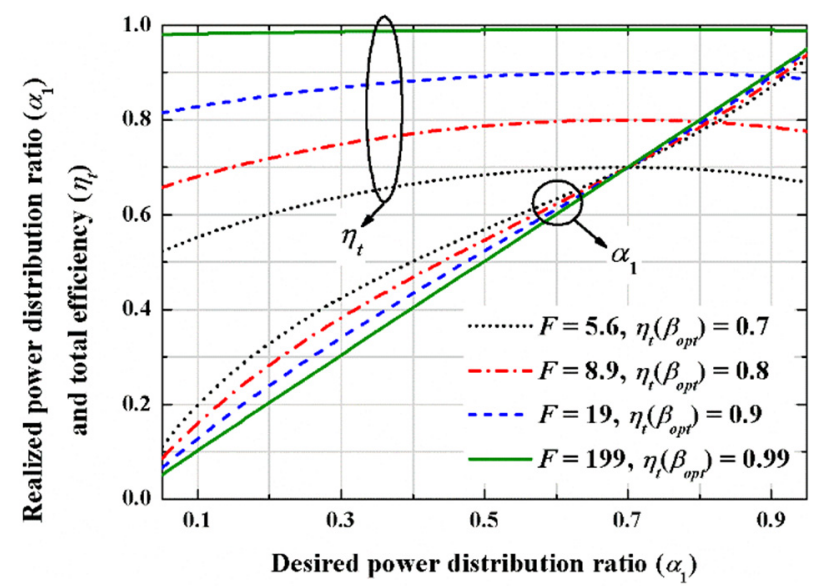

Fig. 5. Realized $\alpha_{1}$ and $\eta_{t}$ as a function of desired $\alpha_{1}$ for different maximum total efficiencies $\eta_{t}\left(\beta_{o p t}\right) \mathrm{s}(17)\left(N=2\right.$ and $\alpha_{1, o p t}=$ $F_{1}^{2} / F^{2}=0.7$ assumed).

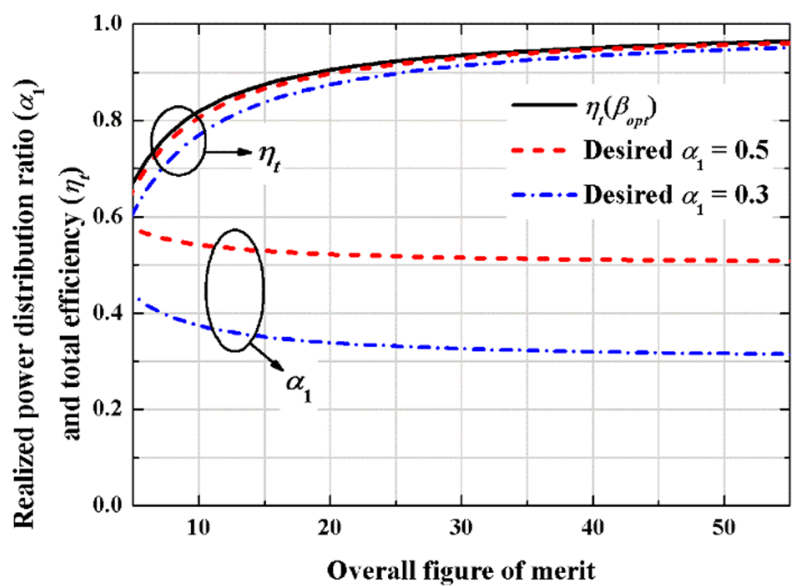

Fig. 6. Realized $\alpha_{1}$ and $\eta_{t}$ as a function of $F$ for different desired $\alpha_{1 \mathrm{~s}}\left(N=2\right.$ and $\alpha_{1, \text { opt }}=F_{1}^{2} / F^{2}=0.7$ assumed $)$.

all cases. Although the accuracy of the realized power distribution ratio slightly decreases as the desired $\alpha_{1}$ deviates from its optimum, the proposed simple solution (21) is good enough for all practical applications. If a higher accuracy is required, the numerical method can be used to obtain the exact solution.

Fig. 6 shows the realized power distribution ratios and total efficiencies as a function of $F(15)$ for different desired $\alpha_{1}$ s of 0.3 and 0.5. The optimum power distribution ratio $\alpha_{1, \text { opt }}$ is fixed at 0.7 . The accuracy of power distribution ratio increases as $F$ increases. This figure also demonstrates that the proposed method is good enough unless $F$ in (15) is extremely small. Notice also that the realized total efficiency $\eta_{t}(12)$ is only slightly lower than its maximum of $\eta_{t}\left(\beta_{\text {opt }}\right)$ (17) obtained when the desired $\alpha_{1}$ is identical to $\alpha_{1, \text { opt }}$ of 0.7 (21).

\section{Validation by Full Wave Electromagnetic Simulation with Three Receivers $(N=3)$}

Fig. 7 shows a configuration of the SIMO WPT system with one transmitter and three receivers for the validation of the proposed solution (21) using full wave EM simulation. The resonant frequencies $\left(f_{0}^{\prime} s\right)$ of $\mathrm{Tx}$ and $\mathrm{Rx}$ loops are $6.78 \mathrm{MHz}$. The

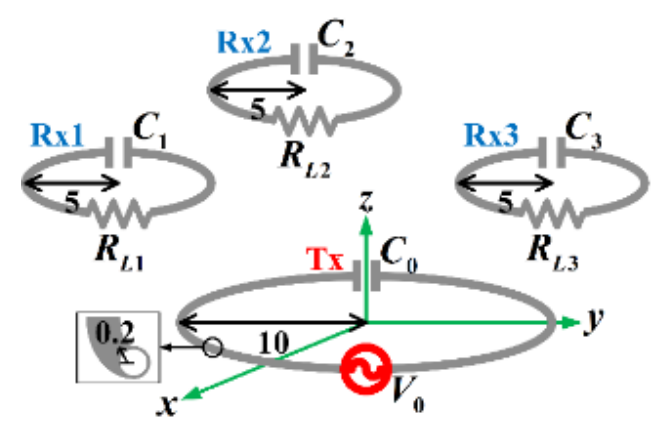

Perspective view

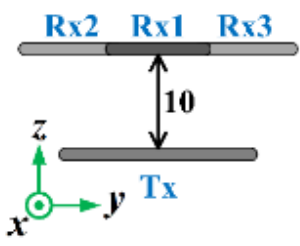

Side view

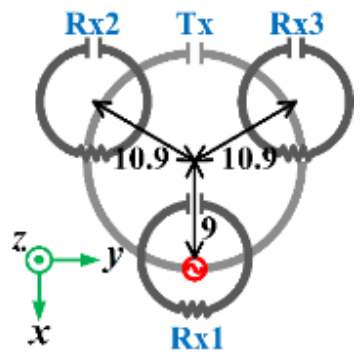

Top view

Fig. 7. A configuration of SIMO WPT system with one transmitter and three receivers for validation by full wave EM simulation (unit: $\mathrm{cm})$. 
Table 1. Element values of Tx and Rx loops $\left(f_{0}=6.78 \mathrm{MHz}\right)$

\begin{tabular}{|c|c|c|c|c|}
\hline & \multicolumn{2}{|c|}{ Tx loop } & \multicolumn{2}{|c|}{ Rx loop } \\
\hline & Theory & Simul. & Theory & Simul. \\
\hline$r(\mathrm{~cm})$ & \multicolumn{2}{|c|}{10} & \multicolumn{2}{|c|}{5} \\
\hline$r_{\text {ring }}(\mathrm{cm})$ & \multicolumn{2}{|c|}{0.2} & \multicolumn{2}{|c|}{0.2} \\
\hline$R(\Omega)$ & 0.034 & 0.035 & 0.017 & 0.017 \\
\hline$L(\mu \mathrm{H})$ & 0.533 & 0.502 & 0.223 & 0.207 \\
\hline$C(\mathrm{nF})$ & 1.034 & 1.097 & 2.472 & 2.657 \\
\hline Q-factor & 668 & 614 & 559 & 506 \\
\hline
\end{tabular}

radii of Tx and Rx loops are $10 \mathrm{~cm}$ and $5 \mathrm{~cm}$, respectively. The radii of the loop ring are $0.2 \mathrm{~cm}$. Theoretical and EM-simulated circuit element values of $\mathrm{Tx}$ and $\mathrm{Rx}$ loops are summarized in Table 1 . The quality factors of the $\mathrm{Tx}$ and $\mathrm{Rx}$ loops based on EM simulations are 614 and 506 at $6.78 \mathrm{MHz}$, respectively.

The center positions of one Tx and three Rx loops in Fig. 7 are $(0 \mathrm{~cm}, 0 \mathrm{~cm}, 0 \mathrm{~cm}),(9 \mathrm{~cm}, 0 \mathrm{~cm}, 10 \mathrm{~cm}),(-5.5 \mathrm{~cm},-9.4 \mathrm{~cm}$, $10 \mathrm{~cm})$, and $(-5.5 \mathrm{~cm}, 9.4 \mathrm{~cm}, 10 \mathrm{~cm})$, respectively. The centers of the Rx loops are $9 \mathrm{~cm}, 10.9 \mathrm{~cm}$, and $10.9 \mathrm{~cm}$ away from the $\mathrm{z}$-axis. The coupling coefficients between two loops can be theoretically evaluated using [17] and can also be extracted using the $S$-parameter obtained from EM simulations [19]. Figures of merit can be obtained using (10). Coupling coefficients and figures of merit between two loops are summarized in Table 2. The EM-simulated $k$ s agree well with the theoretical ones. $F_{1}$, $F_{2}$, and $F_{3}$ are $14.8,10.4$, and 10.4, respectively, using (10). For this example, $\alpha_{1, o p t}=F_{1}^{2} / F^{2}=0.5$ and $\alpha_{2, o p t}=\alpha_{3, o p t}=0.25$ from (19).

Fig. 8(a) and (b) show the necessary load resistances $\left(R_{L i s}\right)$ using (21) based on Tables 1 and 2 to realize a desired power distribution ratio $\left(\alpha_{i} s\right)$ based on the assumptions of $\alpha_{2}=\alpha_{3}$ and $\alpha_{2}=2 \alpha_{3}$, respectively. Note that for each case, $\alpha_{1}+\alpha_{2}+\alpha_{3}=1$. As we can see in (21), $R_{L i}$ decreases as the desired $\alpha_{i}$ increases.

When the desired $\alpha_{1}, \alpha_{2}$, and $\alpha_{3}$ are $0.5,0.25$, and 0.25 in Fig. 8(a), the load resistances $\left(R_{L 1}, R_{L 2}\right.$, and $\left.R_{L 3}\right)$ are the optimum values $\left(R_{L, o p t}\right)(16)$, which are about $0.39 \Omega$.

Table 2. Coupling coefficients and figure of merits between two loops

\begin{tabular}{|c|c|c|c|c|}
\hline & \multicolumn{2}{|c|}{$k_{i}$ or $k_{i j}$} & \multicolumn{2}{|c|}{$F_{i}$ or $F_{i j}$ using $(10)$} \\
\hline & $\begin{array}{c}\text { Theory } \\
\text { [17] }\end{array}$ & Simul. & Theory & Simul. \\
\hline $\mathrm{Tx}-\operatorname{Rx} 1\left(k_{1}, F_{1}\right)$ & \multicolumn{2}{|c|}{0.0265} & 16.2 & 14.8 \\
\hline $\mathrm{Tx}-\operatorname{Rx} 2\left(k_{2}, F_{2}\right)$ & \multicolumn{2}{|c|}{0.0186} & 11.4 & 10.4 \\
\hline $\mathrm{Tx}-\mathrm{Rx} 3\left(k_{3}, F_{3}\right)$ & \multicolumn{2}{|c|}{0.0186} & 11.4 & 10.4 \\
\hline $\mathrm{Rx} 1-\mathrm{Rx} 2\left(k_{12}, F_{12}\right)$ & \multicolumn{2}{|c|}{-0.0071} & -3.95 & -3.96 \\
\hline $\operatorname{Rx} 1-\operatorname{Rx} 3\left(k_{13}, F_{13}\right)$ & \multicolumn{2}{|c|}{-0.0071} & -3.95 & -3.96 \\
\hline $\mathrm{Rx} 2-\mathrm{Rx} 3\left(k_{23}, F_{23}\right)$ & \multicolumn{2}{|c|}{-0.0053} & -2.95 & -2.95 \\
\hline
\end{tabular}

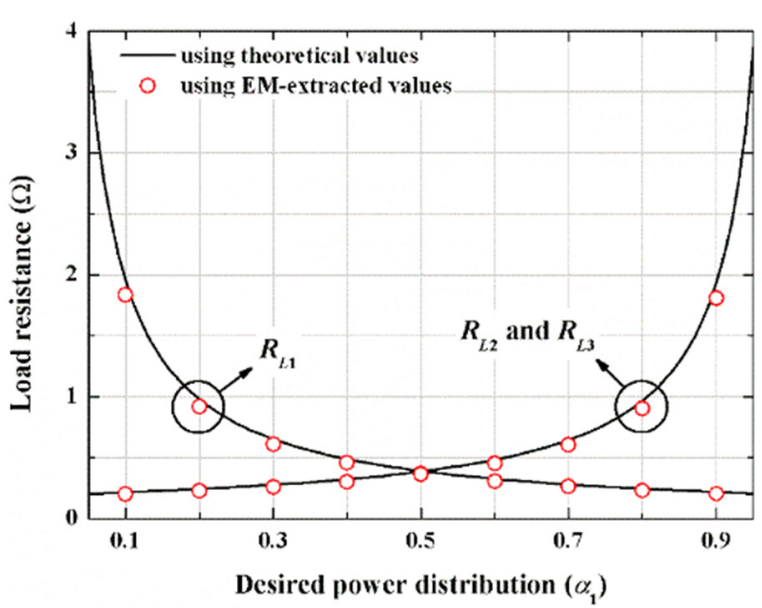

(a)

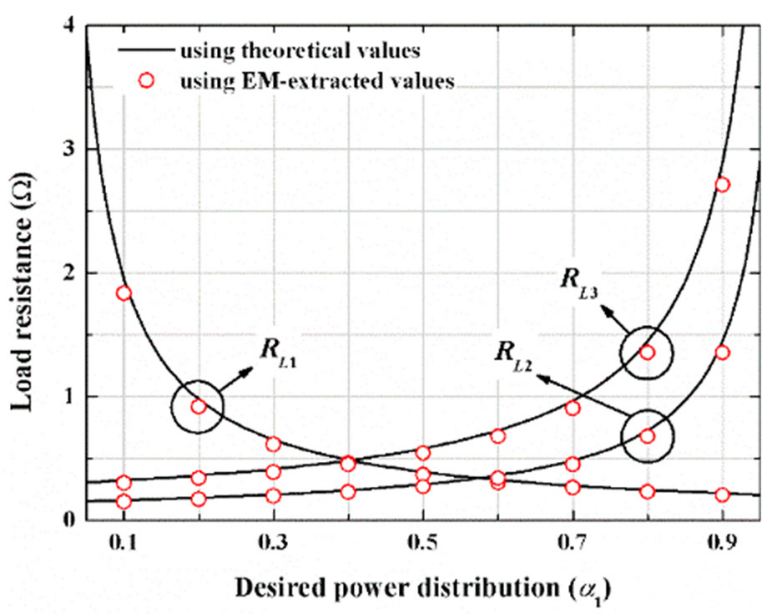

(b)

Fig. 8. Necessary load resistances $\left(R_{L i}=R_{i} \beta_{i}\right.$ for $\left.i=1,2,3\right)$ using (21) based on Tables 1 and 2 as a function of desired $\alpha_{1}\left(f_{0}\right.$ $=6.78 \mathrm{MHz}, N=3, r_{0}=10 \mathrm{~cm}, r_{1}=r_{2}=r_{3}=5 \mathrm{~cm}, r_{\text {ring }}=0.2$ $\mathrm{cm}, \alpha_{1, o p t}=0.5$, and $\alpha_{2, o p t}=\alpha_{3, o p t}=0.25$ ). (a) $\alpha_{2}=\alpha_{3}$ assumed, (b) $\alpha_{2}=2 \alpha_{3}$ assumed.

Fig. 9 shows the realized power distribution ratios and the total system efficiencies of the same SIMO system used in Fig. 8 using theory based on (12) and (18), EM-simulation with $R_{L \text {, }}$, and EM simulation with and additional feeding loop $(50 \Omega)$ [20].

In Fig. 9(a), they have been plotted as a function of the desired $\alpha_{1}$, with $\alpha_{2}=\alpha_{3}$. For this case, the maximum efficiency is $91.6 \%$, and realized power distribution ratios are shown to be almost the same as the desired ones. Again, we can see that the realized $\alpha_{i}$ s agree well with the desired ones for all cases.

Similarly, the realized $\alpha_{i} \mathrm{~s}$ and total efficiency are plotted as a function of the desired $\alpha_{1}$, with $\alpha_{2}=2 \alpha_{3}$ in Fig. 9(b). We can also see that the $\alpha_{i}$ s are well realized, as we desire. For example, when the desired $\alpha_{1}, \alpha_{2}$, and $\alpha_{3}$ are $0.7,0.2$, and 0.1 , respectively, they are realized as $0.69,0.2$, and 0.11 . The results of EM simulations without and with a feeding loop [20] are also shown to be in good agreement with the results of circuit simulations. 


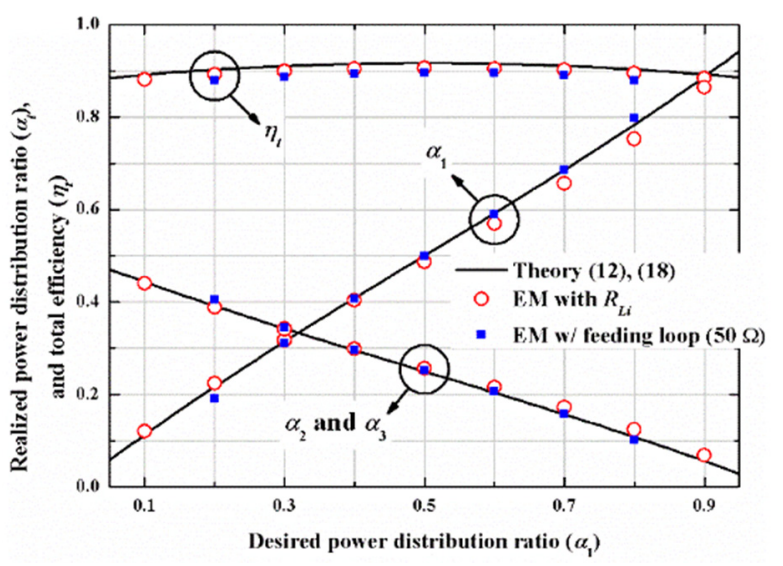

(a)

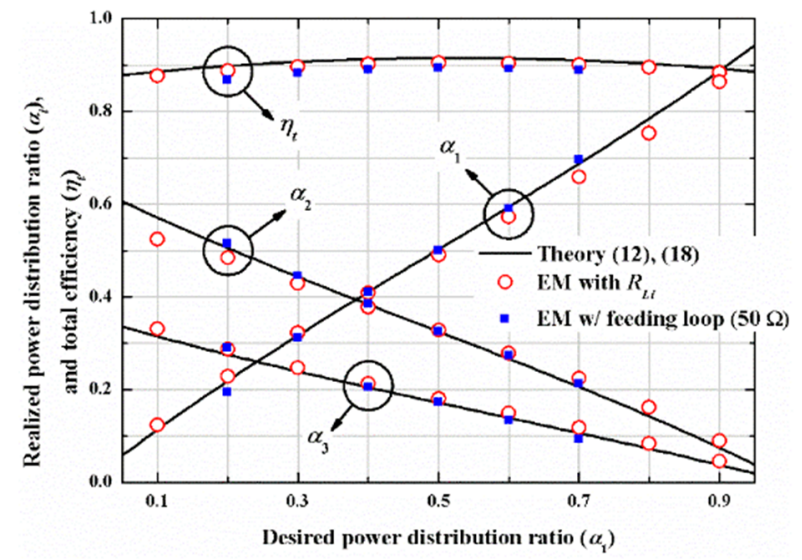

(b)

Fig. 9. Realized $\alpha_{1}$ and $\eta_{t}$ as a function of desired $\alpha_{1}\left(f_{0}=6.78\right.$ $\mathrm{MHz}, N=3, r_{0}=10 \mathrm{~cm}, r_{1}=r_{2}=r_{3}=5 \mathrm{~cm}, r_{\text {ring }}=0.2 \mathrm{~cm}$, $\alpha_{1, o p t}=0.5$, and $\alpha_{2, o p t}=\alpha_{3, o p t}=0.25$ ). (a) $\alpha_{2}=\alpha_{3}$ assumed, (b) $\alpha_{2}=2 \alpha_{3}$ assumed.

Finally in Fig. 10, we show the EM-simulated $\left|H_{z}\right|$ distributions for the cases of desired $\alpha_{1}=0.5$ and $\alpha_{2}=\alpha_{3}=0.25$ and desired $\alpha_{1}=0.7, \alpha_{2}=0.2$, and $\alpha_{3}=0.1$. For these field distributions, a power source of $1 \mathrm{~W}$ was used in HFSS EM simulations. The $\left|H_{z}\right|$ field distributions in Fig. 10(a) and (b) well reflect the desired power distributions or efficiencies. The total efficiencies and power distribution ratios based on theory and EM simulations are in good agreement.

In summary, the realized $\alpha_{i}$ s using the simple closed-form solution (21) show good agreement with the desired ones for a wide range of applications.

\section{CONCLUSION}

In this paper, we analyzed the efficiencies and power distribution ratio among receivers of the SIMO system consisting of multiple receivers. The optimum load for maximum total efficiency was derived using the equivalent circuit. In addition, we obtained the simple formula of the load condition for the desired power distribution ratio. We employed various nu-

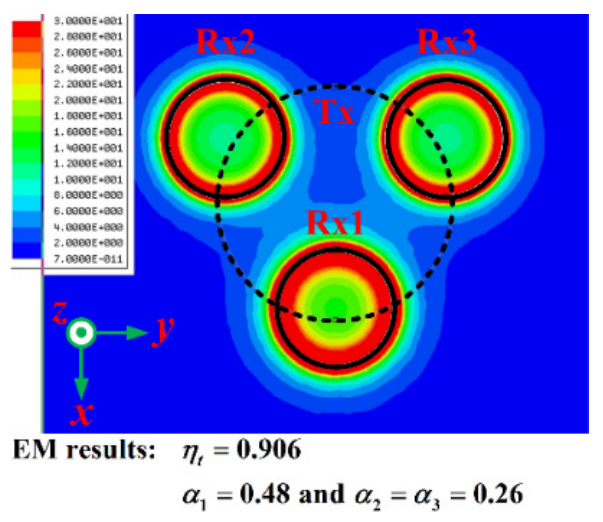

(a)

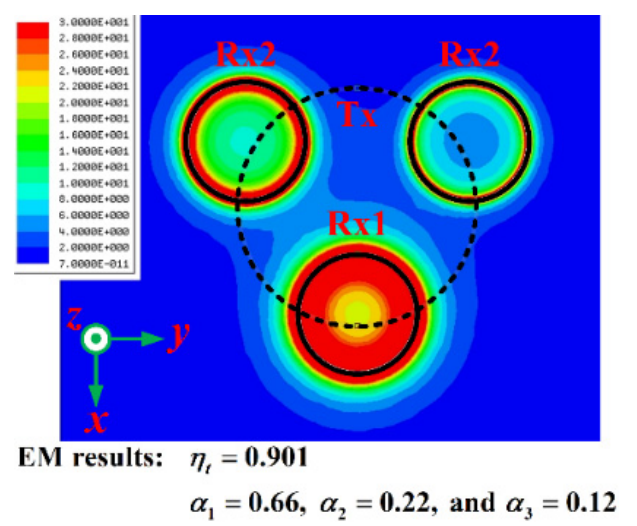

(b)

Fig. 10. EM-simulated $\left|H_{z}\right|$ distribution $\left(f_{0}=6.78 \mathrm{MHz}, N=3\right.$, $r_{0}=10 \mathrm{~cm}, r_{1}=r_{2}=r_{3}=5 \mathrm{~cm}, r_{\text {ring }}=0.2 \mathrm{~cm}, \alpha_{1, o p t}=0.5, \alpha_{2, p p t}$ $=\alpha_{3, o p t}=0.25, \beta_{\text {opt }}=22.9$, and $\left.\eta_{t}\left(\beta_{\text {opt }}\right)=0.916\right)$. (a) Desired $\alpha_{1}=0.5, \alpha_{2}=\alpha_{3}=0.25$, and $\eta_{t}=0.916$, (b) desired $\alpha_{1}=0.7$, $\alpha_{2}=0.2, \alpha_{3}=0.1$, and $\eta_{t}=0.91$.

merical examples and validated the derived formula by comparing the target distribution ratio with the realized one from circuit and EM simulations. The proposed simple formula can also be applied to arbitrary $N$ receivers. There have also been some analytical or closed-form solutions, but their accuracy is far lower than the proposed solution. The proposed formula is expected to be implemented in future SIMO industrial applications due to its simple but relatively accurate property.

This work was supported by Institute for Information \& Communications Technology Promotion (IITP) grant funded by the Korea government (MSIT) (No. IITP-2018-20160-00291, Information Technology Research Center).

\section{REFERENCES}

[1] A. Kurs, A. Karalis, R. Moffatt, J. D. Joannopoulos, P. Fisher, and M. Soljacic, "Wireless power transfer via 
strongly coupled magnetic resonance," Science, vol. 317, no. 5834, pp. 83-86, 2007.

[2] B. H. Choi and J. H. Lee "Design of asymmetrical relay resonators for maximum efficiency of wireless power transfer," International Journal of Antennas and Propagation, vol. 2016, article ID. 8247476, 2016.

[3] G. Kim, T. K. Oh, and B. Lee, "Effects of metamaterial slabs applied to wireless power transfer at $13.56 \mathrm{MHz}$," International Journal of Antennas and Propagation, vol. 2015, article ID 840135, 2015.

[4] Y. Cho, J. J. Kim, D. H. Kim, S. Lee, H. Kim, C. Song, et al., "Thin PCB-type metamaterials for improved efficiency and reduced EMF leakage in wireless power transfer systems," IEEE Transactions on Microwave Theory and Techniques, vol. 64, no. 2, pp. 353-364, 2016.

[5] M. Q. Nguyen, D. Plesa, S. Rao, and J. C. Chiao, "A multiinput and multi-output wireless energy transfer system," in Proceedings of 2014 IEEE MTT-S International Microwave Symposium, Tampa, FL, 2014, pp. 1-3.

[6] S. Kim and B. Lee, "Analysis of efficiencies for multipleinput multiple-output wireless power transfer systems," Journal of Electromagnetic Engineering and Science, vol. 16, no. 2, pp. 126-133, 2016.

[7] A. Kurs, R. Moffatt, and M. Soljacic, "Simultaneous midrange power transfer to multiple devices," Applied Physics Letters, vol. 96, no. 4, article no. 044102, 2010.

[8] E. Bou-Balust, A. P. Hu, and E. Alarcon, "Scalability analysis of SIMO non-radiative resonant wireless power transfer systems based on circuit models," IEEE Transactions on Circuits and Systems I: Regular Papers, vol. 62, no. 10, pp. 2574-2583, 2015.

[9] J. M. Park and S. W. Nam, "Analysis of wireless power transfer characteristics for multiple receivers by time sharing technique," Journal of Electromagnetic Engineering and Science, vol. 11, no. 3, pp. 183-185, 2011.

[10] Y. J. Kim, D. Ha, W. J. Chappell, and P. P. Irazoqui, "Selective wireless power transfer for smart power distribution in a miniature-sized multiple-receiver system," IEEE Transactions on Industrial Electronics, vol. 63, no. 3, pp. 1853-1862, 2016.

[11] M. Fu, T. Zhang, C. Ma, and X. Zhu, "Efficiency and optimal loads analysis for multiple-receiver wireless power transfer systems," IEEE Transactions on Microwave Theory and Techniques, vol. 63, no. 3, pp. 801-812, 2015.

[12] M. R. V. Moghadam and R. Zhang, "Multiuser wireless power transfer via magnetic resonant coupling: performance analysis, charging control, and power region characterization," IEEE Transactions on Signal and Information Processing over Networks, vol. 2, no. 1, pp. 72-83, 2016.

[13] K. E. Koh, T. C. Beh, T. Imura, and Y. Hori, "Impedance matching and power division using impedance inverter for wireless power transfer via magnetic resonant coupling," IEEE Transactions on Industry Applications, vol. 50, no. 3 pp. 2061-2070, 2014.

[14] K. Lee and D. H. Cho, "Analysis of wireless power transfer for adjustable power distribution among multiple receivers," IEEE Antennas and Wireless Propagation Letters, vol. 14, pp. 950-953, 2015.

[15] M. Fu, H. Yin, and C. Ma, "Megahertz multiple-receiver wireless power transfer systems with power flow management and maximum efficiency point tracking," IEEE Transactions on Microwave Theory and Techniques, vol. 65, no. 11, pp. 4285-4293, 2017.

[16] M. Liu, M. Fu, Y. Wang, and C. Ma, "Battery cell equalization via megahertz multiple-receiver wireless power transfer," IEEE Transactions on Power Electronics, vol. 33, no. 5, pp. 4135-4144, 2017.

[17] G. Kim and B. Lee, "Alternative expressions for mutual inductance and coupling coefficient applied in wireless power transfer," Journal of Electromagnetic Engineering and Science, vol. 16, no. 2, pp. 112-118, 2016.

[18] G. Kim and B. Lee, "Analysis of magnetically coupled wireless power transfer between two resonators based on power conservation," in Proceedings of 2014 IEEE Wireless Power Transfer Conference, Jeju, Korea, 2014, pp. 231-234.

[19] C. J. Kim and B. Lee, "Analysis of magnetically coupled wireless power transmission for maximum efficiency," Journal of Electromagnetic Engineering and Science, vol. 11, no. 3, pp. 156-160, 2011.

[20] M. Kiani, U. M. Jow, and M. Ghovanloo, "Design and optimization of a 3-coil inductive link for efficient wireless power transmission," IEEE Transactions on Biomedical Circuits and Systems, vol. 5, no. 6, pp. 579-591, 2011. 


\section{Gunyoung Kim}

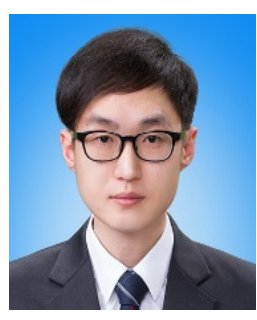

received a B.S. degree in Radio Communication Engineering from Kyung Hee University, Yongin, Korea in 2010, an M.S. degree in Electronics and Radio Engineering from Kyung Hee University in 2012, and a Ph.D. degree in Electronics Engineering from Kyung Hee University in 2018. Currently, he is a post-doctor in the College of Electronics and Information at Kyung Hee University. His fields of research include microwave antennas, passive devices, wireless power transmission, and metamaterials.

\section{Seunghyun Boo}

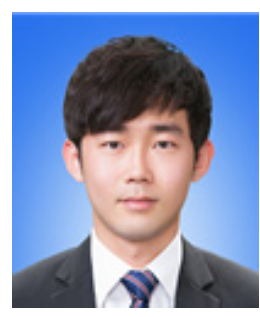

received a B.S. in Electronics Engineering from Kyung Hee University, Yongin, Korea, in 2017. He is currently working toward an M.S. in Electronics Engineering from Kyung Hee University. His fields of research include microwave antennas, passive devices, and wireless power transmission.

\section{Sanghoek Kim}

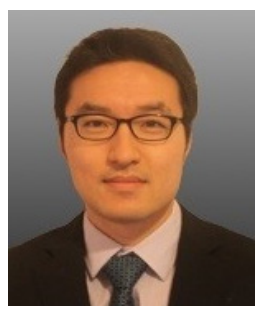

received B.S. degree with a double major in Electrical Engineering and Mathematics from Seoul National University, Seoul, Korea in 2007 and the M.S. and Ph.D. degrees in electrical engineering from Stanford University, CA, USA in 2013. His research focuses on applications of radio-frequency technology and electromagnetics theory in wireless power transfer, communications, and signal integrity in high-speed channels. After graduating, he worked at Qualcomm Inc. as a signal/power integrity engineer and at $\mathrm{SiBeam}$, Inc. as a mmWave system engineer. Currently, he is an assistant professor in the Department of Electronics Engineering at Kyung Hee University.

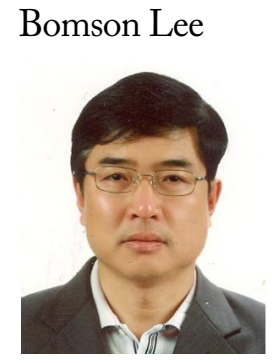

received a B.S. degree in Electrical Engineering from Seoul National University, Seoul, Korea, in 1982. From 1982 to 1988 , he was with the Hyundai Engineering Company Ltd., Seoul, Korea. He received M.S. and Ph.D. degrees in Electrical Engineering from the University of Nebraska, Lincoln, NE, USA in 1991 and 1995, respectively. In 1995, he joined the faculty at Kyung Hee University, where he is currently a professor in the Department of Electronics Engineering. From 2007 to 2008, he was the chair of the technical group for microwave and radio wave propagation in the Korea Institute of Electromagnetic Engineering \& Science (KIEES). In 2010, he was Editor-inChief of the Journal of the Korean Institute of Electromagnetic Engineering and Science. From 2015 to 2016, he was vice-chairman of KIEES. In 2017, he was executive vice-chairman of KIEES. Since 2018, he has been president of KIEES. His research interests include microwave antennas, RF identification (RFID) tags, microwave passive devices, wireless power transfer, and metamaterials. 